\title{
ON LINEARIZATION OF THE KLEIN-GORDON EQUATION BY NONTRADITIONAL METHODS
}

\author{
(Presented by H. Keres)
}

In the present paper two nontraditional methods of the linearization of the KleinGordon equation are considered. It is shown that a generalized Dirac equation obtained recently by J. P. Gazeau [ $\left.{ }^{1}\right]$ is equivalent to the Hurley equation $\left.{ }^{[2}\right]$. Next, such linearization is made by using the Kemmer-Duffin spin- 0 formalism $\left[{ }^{3}\right]$.

1. According to E. P. Wigner, to every invariant quantum-mechanical system of equations corresponds a unitary continuous representation of the Poincaré group $[4,5]$. It is known that irreducible representations describe elementary systems. Every irreducible representation is determined by the eigenvalues of two invariants - the square of the momentum operator $P^{2}$ and the square of the Pauli-Lubanski pseudovector $w^{2}-m^{2}$ and $s(s-1-$ $+1) m^{2}$, respectively. Let the irreducible representation defined by these eigenvalues be denoted $\mathfrak{T}_{1.3}\left(\mathrm{~m}^{2}, s\right) \quad\left[{ }^{5-8}\right]$. If $m^{2}>0$, the operator of the sign of the energy $\hat{E}=P^{0}\left|P^{0}\right|^{-1}$ is also an invariant. Since the representations of this kind possess a physical meaning, we confine ourselves to the representations with $m^{2}>0$. Now, for every element $\Phi$ of the space of a given representation $\mathfrak{T}_{1.3}\left(\mathrm{~m}^{2}>0, s\right)$ one can write

$$
\begin{aligned}
& P^{2} \Phi(p, \sigma)=m^{2} \Phi(p, \sigma), \\
& w^{2} \Phi(p, \sigma)=-s(s+1) m^{2} \Phi(p, \sigma), \\
& \hat{E} \Phi(p, \sigma)=\varepsilon \Phi(p, \sigma),
\end{aligned}
$$

where $p_{\mu}$ is 4 -momentum, $\sigma=-s,-s+1, \ldots, s$ and $\varepsilon= \pm 1$. In case of $m^{2}>0$, the little group is $\mathrm{SO}_{3}$ and $\Phi(p, \sigma)$ is the spinor of the rank $2 s-1-1$.

The linearization of equation (1) in a general case leads to an increase of the dimension. The Harish-Chandra first-order relativistically invariant equation $\left[{ }^{9}\right]$ of the following form can be considered as a linearized form of the Klein-Gordon equation:

$$
\begin{aligned}
& \left(P^{\mu} \beta_{\mu}-m\right) \psi=0, \\
& {\left[\beta_{\mu}, M_{\rho \sigma}\right]=i\left(g_{\mu \rho} \beta_{\sigma}-g_{\mu \sigma} \beta_{\rho}\right),} \\
& \sum_{(P)} \beta_{\alpha} \ldots \beta_{\gamma}\left(\beta_{\rho} \beta_{\sigma}-g_{\rho \sigma}\right)=0 .
\end{aligned}
$$

Here $(4 c)$ is the sum over all the permutations of the indices $\alpha, \ldots, \gamma$, $\mathrm{Q}, \sigma$. Well-known equations of that type are the Kemmer-Duffin equation for spin 0 and 1 , and the Dirac equation for spin $1 / 2$.

2. Recently J.P. Gazeau linearized the Klein-Gordon equation by using rectangular matrices, obtaining the so-called generalized. Dirac equation for single mass and for an arbitrary single spin. We add to this linearization the requirement that the matrices should be of minimum dimensions. 
Thus, generally rectangular matrices $Z_{\mu}, Y_{\mu}$ with minimum dimensions are sought, so that the equation

$$
P^{\mu} P^{v} Z_{\mu} Y_{v} \Phi=m^{2} \Phi
$$

would be equivalent to the Klein-Gordon equation (1). If such matrices are found, then, defining the new wavefunction

$$
\psi=\left|\begin{array}{l}
\psi_{1}=m^{-1} P^{\mu} Y_{\mu} \Phi \\
\psi_{0}=\Phi
\end{array}\right|
$$

one can see that the system

$$
\begin{array}{r}
-m \psi_{1}+P^{\mu} Y_{\mu} \psi_{0}=0, \\
P^{\mu} Z_{\mu} \psi_{1}-m \psi_{0}=0
\end{array}
$$

is equivalent to equation (5) and can be taken in the form of (4a)

$$
\left(P^{\mu} \Gamma_{\mu}-m\right) \psi=0 \text {, }
$$

where

$$
\Gamma_{\mu}=\left|\begin{array}{cc}
0 & Y_{\mu} \\
Z_{\mu} & 0
\end{array}\right|
$$

Equation (5) is satisfied by $4 s \times(2 s+1)$-dimensional matrices

$$
\begin{aligned}
Y_{\varepsilon}^{0} & =a_{2} \tau_{0}(s), \\
Y_{\varepsilon}^{j} & =\varepsilon a_{2} s^{-1}\left(-\tau_{0}(s) M^{j}(s)+b_{2} t(s) K^{j}(s)\right)
\end{aligned}
$$

and by $(2 s+1) \times 4 s$-dimensional matrices

$$
\begin{aligned}
& Z_{\varepsilon}^{0}=a_{1} \tau_{0}^{+}(s), \\
& Z_{\varepsilon}^{j}=-\varepsilon a_{1} s^{-1}\left(-M^{j}(s) \tau_{0}^{+}(s)+b_{1} K^{j}(s)+t^{+}(s)\right),
\end{aligned}
$$

where $a_{1} a_{2}=b_{1} b_{2}=1, \varepsilon= \pm 1$ and $j=1,2,3$. The matrices $\tau_{\varepsilon}^{\mu}(s), M^{j}(s)$, $t(s)$ and $K^{j}(s)$ are given in the appendices A, B, C. Indeed, equation (5) is equivalent to (1) because of the relation

$$
Z_{\varepsilon}^{\mu}(s) \underset{\varepsilon}{Y_{\varepsilon}^{v}}(s)=g^{\mu \nu}-i s^{-1} M_{\varepsilon}^{\mu v}
$$

If the parameters have the values $a_{1}=b_{1}=a_{2}=b_{2}=1$, the $(6 s+1)$ dimensional matrices $\Gamma_{\varepsilon}^{\mu}$ take the form [ $\left.{ }^{1}\right]$

$$
\Gamma_{\varepsilon}^{\mu}=\left|\begin{array}{cc}
0 & \tau_{\varepsilon}^{\mu} \\
\tilde{\tau}_{\varepsilon}^{\mu+} & 0
\end{array}\right| .
$$

After the unitary transformation (see App. C)

$$
V=\left|\begin{array}{ccc}
0 & \tau^{0}(s) & t(s) \\
1 & 0 & 0
\end{array}\right|,
$$

one gets easily

$$
\Gamma_{\varepsilon}^{\mu} \rightarrow \beta_{\varepsilon}^{\mu}=V^{+} \Gamma_{\varepsilon}^{\mu} V=\left|\begin{array}{ccc}
0 & \tilde{\tau}_{\varepsilon}^{\mu+}(s) \tau_{\varepsilon}^{0}(s) & \tilde{\tau}_{\varepsilon}^{\mu}(s) t(s) \\
\tau_{\varepsilon}^{0+}(s) \tau_{\varepsilon}^{\mu}(s) & 0 & 0 \\
t^{+}(s) \tau_{\varepsilon}^{\mu}(s) & 0 & 0
\end{array}\right|
$$


Thus we have shown that the linearized form $(6)-(8)$ of second-order equation (1) (or (5)) is the Hurley equation $\left[{ }^{2}\right]$.

In conclusion, starting with an irreducible representation $\mathfrak{T}_{1.3}\left(\mathrm{~m}^{2}>\right.$ $>0, s$ ), using Gazeau's method of linearization and demanding that the dimensions of the matrices be minimal, the Hurley relativistically invariant equation for the single mass $m$ and the single spin $s$ can be obtained. The latter equation corresponds to the interlocking scheme $(s, 0) \sim(s-1 / 2,1 / 2)$ and satisfies $(4 \mathrm{c})$ in case $\beta_{0}{ }^{3}=\beta_{0}\left[{ }^{9,10}\right]$.

Next we consider a relativistically invariant equation with the interlocking scheme $(s, 0) \sim(s+1 / 2,1 / 2)$, which we take in the form of (6), (7). Since the representation $(s+1 / 2,1 / 2)$ can be obtained from $(s-1 / 2,1 / 2)$ by the substitution $s \rightarrow s+1$, the corresponding $\Gamma^{\mu}-s$ are of greater dimensions. $\Gamma^{\mu}-s$ comprise now the $4(s-1) \times(2 s+1)$. dimensional matrices (see App. D)

$$
\begin{aligned}
Y_{\varepsilon}^{0} & =a_{2} t(s+1), \\
Y_{\varepsilon}^{j} & =\varepsilon a_{2}(s+1)^{-1}\left(t(s+1) M^{j}(s)+b_{2} \tau_{\varepsilon}^{0}(s+1) K^{j+}(s+1)\right)
\end{aligned}
$$

and the $(2 s+1) \times 4(s+1)$-dimensional matrices

$$
\begin{aligned}
& Z_{\varepsilon}^{0}=a_{1} t^{+}(s+1), \\
& Z_{\varepsilon}^{j_{i}}=-\varepsilon a_{1}(s+1)^{-1}\left(M^{j}(s) t^{+}(s+1)+b_{1} K^{j}(s+1) \tau_{\varepsilon}^{0+}(s+1)\right),
\end{aligned}
$$

where $a_{1} a_{2}=b_{1} b_{2}=1, \varepsilon= \pm 1$.

Since now

$$
Z_{\varepsilon}^{\mu} Y_{\varepsilon}^{v}=g^{\mu \nu}+i(s+1)^{-1} M_{\varepsilon}^{\mu \nu} .
$$

Equation (5) is indeed equivalent to equation (1). At the unitary transformation

$$
V=\left|\begin{array}{ccc}
0 & t(s+1) & \tau^{0}(s+1) \\
1 & 0 & 0
\end{array}\right|
$$

$\Gamma_{\varepsilon}{ }^{\mu}$ transform in the following way

$$
\Gamma_{\varepsilon}^{\mu} \rightarrow \beta_{\varepsilon}^{\mu}=\left|\begin{array}{ccc}
0 & a_{1}\left(g^{0 \mu}-\varepsilon(s+1)^{-1} M^{\mu}(s)\right) & -\varepsilon a_{1} b_{1} K^{\mu}(s+1) \\
a_{2}\left(g^{0 \mu}+\varepsilon(s+1)^{-1} M^{\mu}(s)\right) & 0 & 0 \\
\varepsilon a_{2} b_{2} K^{\mu+}(s+1) & 0 & 0
\end{array}\right| .
$$

In case $a_{1}=a_{2}=b_{1}=b_{2}=1$ one can see that

$$
\begin{aligned}
\Gamma_{\varepsilon}^{\mu}=\left|\begin{array}{cc}
0 & \mathrm{\varrho}_{\varepsilon}^{\mu} \\
\tilde{\mathrm{Q}_{\varepsilon}^{\mu}} & 0
\end{array}\right| \rightarrow \beta_{\varepsilon}^{\mu}= \\
=\left|\begin{array}{ccc}
0 & g^{\mu 0}-\varepsilon(s+1)^{-1} M^{\mu}(s) & \varepsilon K^{\mu}(s+1) \\
g^{\mu 0}+\varepsilon(s+1)^{-1} M^{\mu}(s) & 0 & 0 \\
-\varepsilon K^{\mu+}(s+1) & 0 & 0
\end{array}\right| .
\end{aligned}
$$

It follows from the explicit form of $\beta_{\varepsilon}^{\mu}$ (13a) that the equation

$$
\left(P_{\mu} \beta_{\varepsilon}^{\mu}-m\right) \psi_{\varepsilon}=0
$$

is a Harish-Chandra equation, and it satisfies minimal equation (4c) in case $\beta_{0}{ }^{3}=\beta_{0}$. After calculating the generators corresponding to equation (13), one can see that in case $\varepsilon=1$, the equation obtained has the 
wanted interlocking scheme $(s, 0) \sim(s-1 / 2,1 / 2)$ and in case $\varepsilon=-1$ the scheme $(0, s) \sim(1 / 2, s+1 / 2)$.

One of the varieties of this equation is the Kemmer-Duffin spin-0 equation, but the interlocking scheme $(s, 0) \sim(s-1 / 2,1 / 2)$ is connected with the Kemmer-Duffin spin-1 equation.

If the minimal electromagnetic interaction is present, equation (5) with matrices (8) leads to the Pauli-Waerden equation [ [ $\left.{ }^{1}\right]$

$$
\left(D^{2}-q(2 s)^{-1} F_{\mu \nu} M_{\varepsilon}^{\mu \nu}-m^{2}\right) \Phi_{\varepsilon}=0,
$$

where $D_{\mu}=P_{\mu}-q A_{\mu}$ and $F_{\mu \nu}=i q^{-1}\left[D_{\mu}, D_{v}\right]$.

W. J. Hurley has shown in $\left[{ }^{2}\right]$ that this equation is causal and equivalent to the system of equations which are obtained by introducing the minimal electromagnetic interaction into linearized equations $(6)-(8)$. form

In case of interaction, equation (5) with matrices (11) takes the

$$
\left(D^{2}+q(2(s+1))^{-1} F_{\mu v} M_{\varepsilon}^{\mu v}-m^{2}\right) \Phi_{\varepsilon}=0 .
$$

It is shown in [ ${ }^{11}$ ] that this equation is also causal and equivalent to the system of equations obtainable from equation (13). Though the interlocking schemes $(s, 0) \sim(s-1 / 2,1 / 2)$ and $(s, 0) \sim(s+1 / 2,1 / 2)$ are quite similar to each other, the solutions of the corresponding equations are different, for the interaction terms are different.

3. Secondly, we deal with the linearization of the Klein-Gordon equation by means of the Kemmer-Duffin spin-0 algebra $\left[{ }^{3}\right]$. For this purpose we define the wavefunction

where

$$
\psi=\Delta \otimes \Phi
$$

$$
\Delta_{A}=\left\{\begin{array}{l}
m^{-1} P_{\mu}, A=\mu=0,1,2,3 \\
1, A=5
\end{array}\right.
$$

Now the system

$$
\begin{array}{r}
P^{\mu} \psi_{\mu}-m \psi_{5}=0, \\
\psi_{\mu}-m^{-1} P_{\mu} \psi_{5}=0
\end{array}
$$

arising in the stead of equation (1), can be presented in a matrix form as a relativistically invariant equation

$$
\left(P_{\mu} \beta^{\mu}(s)-m\right) \psi=0,
$$

where $\beta^{\mu}(s)=\beta^{\mu} \otimes I(s)$ and $\beta^{\mu}$ generate the Kemmer-Duffin spin-0 algebra $\left[{ }^{12,13}\right]$.

Equation (16) follows from a scalar Lagrangian, Indeed, since the representation $S_{1,3}\left(m^{2}>0,0\right) \otimes T_{\varepsilon}$ is equivalent to the representation $\mathfrak{T}_{1.3}\left(m^{2}>0,0\right) \otimes T_{-\varepsilon}$ due to relation $\left[{ }^{8}\right]$

$$
T_{\varepsilon}^{+}(\Lambda) S(\Lambda p) T_{\varepsilon}(\Lambda)=S(p)
$$

the quantity

$$
\psi_{\rho}^{+}(p) S(p) \Phi_{\varepsilon}(p) \equiv \psi_{-\varepsilon}^{+}(p) \Phi_{\varepsilon}(p)
$$

proves to be a scalar. Here $T_{\varepsilon}= \begin{cases}T^{(k, l)}, & \varepsilon=1, \\ T^{(l, k)}, & \varepsilon=-1,\end{cases}$

is an irreducible representation of the Lorentz group and $S(p)$ is the Hermitian matrix, by which the inner product, with respect to which the 
representation $\mathfrak{T}_{1.3}\left(m^{2}>0,0\right) \otimes T_{\varepsilon}$ is unitary, is constructed. The Lagrangian mentioned above has the form

$$
\mathfrak{L}_{\varepsilon}=2^{-1} \bar{\psi}_{-\varepsilon} \beta^{\mu}(s)\left(P_{\mu} \psi_{\varepsilon}\right)-2^{-1}\left(P_{\mu} \bar{\psi}_{-\varepsilon}\right) \beta^{\mu}(s) \psi_{\varepsilon}-m \bar{\psi}_{-\varepsilon} \psi_{\varepsilon},
$$

where $\bar{\psi}_{\varepsilon}=\psi_{\varepsilon}^{+} \bar{g}(s), \psi_{-\varepsilon}=S(P) \psi_{\varepsilon}$ and $\bar{g}(s)=\bar{g} \otimes I(s)$. The representations of the Lorentz group corresponding to equation (16) are

$$
\begin{aligned}
& \mathfrak{C}_{+}=((1 / 2,1 / 2) \oplus(0,0)) \otimes(s, 0)=(s+1 / 2,1 / 2) \oplus(s-1 / 2,1 / 2) \oplus(s, 0), \\
& \mathfrak{C}_{-}=((1 / 2,1 / 2) \oplus(0,0)) \otimes(0, s)=(1 / 2, s+1 / 2) \oplus(1 / 2, s-1 / 2) \oplus(0, s) .
\end{aligned}
$$

The relativistically invariant equations with these interlocking schemes have been treated in detail by W. J. Hurley [ $\left.{ }^{14}\right]$.

For the linearization of the Pauli-Wàerden equation it is convenient to define a new wavefunction

where

$$
\psi_{\mathrm{e}}=\mathfrak{D} \otimes \Phi_{\mathrm{e}},
$$

$$
\mathfrak{D}_{A}= \begin{cases}m^{-1} D_{\mu}, & A=\mu=0,1,2,3 ; \\ 1, & A=5 .\end{cases}
$$

The system corresponding to (14) or (15)

$$
\begin{gathered}
D_{\mu} \psi_{\varepsilon}^{\mu}+\left(b F_{\mu \nu} \beta_{5} \otimes M_{\varepsilon}^{\mu v}-m\right) \psi_{\varepsilon}^{5}=0, \\
\psi_{\varepsilon}^{\mu}-m^{-1} D^{\mu} \psi_{\varepsilon}^{5}=0
\end{gathered}
$$

can be presented by the use of the matrices $\beta^{\mu}(s)$ in the form

$$
\left(D_{\mu} \beta^{\mu}(s)+b F_{\mu \nu} \beta_{5} \otimes M_{\varepsilon}^{\mu v}-m\right) \psi_{\varepsilon}=0 .
$$

This is a relativistically invariant equation with the interlocking scheme $\boldsymbol{C}_{\mathrm{e}}$.

Equation (17) can be obtained from the Lagrangian

$$
\mathcal{L}=\sum_{\varepsilon= \pm 1}\left\{2^{-1} \bar{\psi}-\varepsilon \beta^{\mu}(s)\left(D_{\mu} \psi_{\varepsilon}\right)-2^{-1}\left(\bar{D}_{\mu} \bar{\psi}_{-\varepsilon}\right) \beta^{\mu}(s) \psi_{\varepsilon}+b \bar{\psi}-\varepsilon \Theta_{\varepsilon} \psi_{\varepsilon}-m_{\bar{\psi}-\varepsilon} \psi_{\varepsilon}\right\} .
$$

Here we have denoted $\bar{\psi}_{\varepsilon}=\psi_{\varepsilon}^{+} \bar{g}(s), \widetilde{D}_{\mu}=P_{\mu}+q A_{\mu}$ and $\Theta_{\varepsilon}=F_{\mu \nu} \beta_{5} \otimes M_{\varepsilon}^{\mu \nu}$.

Indeed, varying $\mathfrak{L}$ with respect to $\psi_{\varepsilon}, \bar{\psi}_{-\varepsilon}$ as independent co-ordinates, the Euler-Lagrange equations will be

$$
\begin{gathered}
\left(\beta^{\mu}(s) D_{\mu}+b \Theta_{\varepsilon}-m\right) \psi_{\varepsilon}=0, \\
\left(\bar{D}_{\mu} \bar{\psi}_{-\varepsilon}\right) \beta^{\mu}(s)-b \bar{\psi}_{-\varepsilon} \Theta_{\varepsilon}+m \bar{\psi}_{-\varepsilon}=0 .
\end{gathered}
$$

The respective Hamiltonian takes the form

$$
\mathcal{H}=\sum_{\varepsilon} \bar{\psi}_{-\varepsilon}\left(-\beta_{j}(s) D^{j}+q \beta_{0}(s) A^{0}-b \Theta_{\varepsilon}-m\right) \psi_{\varepsilon} .
$$

As was shown in $\left[{ }^{15}\right]$, one can separate from $\psi_{e}$ its "physical» part $\psi_{\mathrm{e}}^{(+)}=$ $=\Pi^{(+)}(s) \psi_{\varepsilon}$ by making use of the projection operator $\Pi^{(z)}(s)=\Pi^{(\Xi)} \otimes I(s)$ (see App. E). The remaining part of $\psi_{\varepsilon}$ may be expressed from (17) (19) through the «physical» part as follows -

$$
\begin{aligned}
& \psi_{\varepsilon}^{(-)}=m^{-1} \beta^{j}(s) D_{j} \psi_{\varepsilon}^{(+)}, \\
& \bar{\psi}_{-\varepsilon}^{(-)}=-m^{-1}\left(\bar{D}^{j} \bar{\psi}_{-\varepsilon}^{(+)}\right) \beta_{j}(s) .
\end{aligned}
$$


After eliminating superfluous eigenvalues, the Hamiltonian will contain only the «physical» part

$$
\begin{gathered}
\Im C=\Sigma \bar{\psi}_{-\varepsilon}^{(+)}\left(-m^{-1} \beta^{0}(s) \beta^{i}(s) \beta^{j}(s) D_{i} D_{j}+q \beta^{0}(s) \beta^{0}(s) A_{0}-\right. \\
\left.-b \beta^{0}(s) \Theta_{\varepsilon}+m \beta^{0}(s)\right) \psi_{\varepsilon}^{(+)} .
\end{gathered}
$$

On account of the relation $\Pi^{(+)} \beta_{i} \beta_{j} \Pi^{(+)}=g_{i j} \beta_{5}$, the eigenvalues $\lambda$ of $\pi$ can be calculated from the equation

$$
\left\{\left(q A^{0}-\lambda\right)^{2}-\vec{D}^{2}+2 m b(-i \varepsilon \vec{E}+\vec{B}) \vec{M}(s)-m^{2}\right\}_{\varphi_{\varepsilon}}=0,
$$

where $\vec{E}$ and $\vec{B}$ are the electric and magnetic fields, respectively.

It is a pleasure to thank M. Kōiv for several useful discussions.

\section{Appendix A}

The generators $M^{\mu}(s)=M^{\mu+}(s)\left(M^{0} \equiv 0\right)$ of the irreducible representation $\mathrm{O}_{3}(s)$ of the rotation group $\mathrm{O}_{3}$ are defined as

$$
\begin{aligned}
& M^{1}(s)_{q t}=-1 / 2\left\{\sqrt{(2 s-q)(q+1)} \delta_{q+1, t}+\sqrt{(2 s-q+1) q} \delta_{q-1, t}\right\}, \\
& M^{2}(s)_{q t}=i / 2\left\{\sqrt{(2 s-q)(q+1)} \delta_{q+1, t}-\sqrt{(2 s-q+1) q} \delta_{q-1, t}\right\}, \\
& M^{3}(s)_{q t}=-(s-q) \delta_{q t} \quad(q, t=0,1, \ldots, 2 s),
\end{aligned}
$$

which satisfy the commutation rule

$$
\begin{aligned}
& {\left[M^{i}, M^{j}\right]=i \varepsilon_{k}^{0 i j} M^{k}} \\
& \left(\varepsilon^{0123}=1\right) .
\end{aligned}
$$

The generators of the irreducible representation of the Lorentz group $O_{\varepsilon}=\left\{\begin{array}{l}O_{1,3}(s, 0), \quad \varepsilon=1 \\ O_{1,3}(0, s), \varepsilon=-1\end{array}\right.$ can be expressed through the generators (A.1).

$$
M_{\varepsilon}^{\mu v}=-\varepsilon i\left(g^{0 \mu} M^{v}(s)-g^{0 v} M^{\mu}(s)\right)+\varepsilon_{\alpha}^{0 \mu v} M^{\alpha}(s) .
$$

\section{Appendix B}

Let us have $2(2 s+1)$-dimensional one-column matrices

$$
\xi_{a q}(s)=e_{q}(s) \otimes e_{a}(1 / 2),
$$

where the $(2 s+1)$-dimensional one-column matrices have the form

$$
\left(e_{q}(s)\right)_{t}=\delta_{q t}, \quad q, t=0,1, \ldots, 2 s .
$$

By means of (B.1) one can define $4 s \times(2 s+1)$-dimensional matrices

$$
\begin{aligned}
& \left(\tau_{\varepsilon}^{0}(s)\right)_{q}=(2 s)^{-1 / 2}\left(\sqrt{q} \xi_{1 q}+\sqrt{2 s-q} \xi_{2 q}\right), \\
& \left(\tau_{\varepsilon}^{1}(s)\right)_{q}=\varepsilon(2 s)^{-1 / 2}\left(-\sqrt{2 s-q} \xi_{1, q+1}-\sqrt{q} \xi_{2, q-1}\right), \\
& \left(\tau_{\varepsilon}^{2}(s)\right)_{q}=\varepsilon(2 s)^{-1 / 2}\left(-\sqrt{2 s-q} \xi_{1, q+1}+\sqrt{q} \xi_{2, q-1}\right), \\
& \left(\tau_{\varepsilon}^{3}(s)\right)_{q}=\varepsilon(2 s)^{-1 / 2}\left(\sqrt{q} \xi_{1 q}-\sqrt{2 s-q} \xi_{2 q}\right) \\
& (\varepsilon= \pm 1),
\end{aligned}
$$


which satisfy the relations

$$
\begin{aligned}
& \tilde{\tau}_{\varepsilon}^{\mu+} \tau_{\varepsilon}^{v}=g^{\mu v}-i S^{-1} M_{\varepsilon}^{\mu v}, \\
& \tau_{\varepsilon}^{0+}\left(\tilde{\tau}_{\varepsilon}^{\mu} \tau_{\varepsilon}^{v+}-\tilde{\tau}_{\varepsilon}^{v} \tau_{\varepsilon}^{\mu+}\right) \tau_{\varepsilon}^{0}= \\
& =-2 \varepsilon S^{-1}\left(g^{0 \mu} M^{v}-g^{0 v} M^{\mu}\right)-\frac{i}{S^{2}} \varepsilon_{\alpha}^{0 \mu v} M^{\alpha} .
\end{aligned}
$$

Here $\tilde{\tau}_{\varepsilon}^{\mu}=g^{\mu \mu} \tau_{\varepsilon}^{\mu}=\tau_{-\varepsilon}^{\mu}$ and $g_{\mu \nu}$ denotes the metric tensor of the Minkowski space with $g=\operatorname{diag}(+---)$.

Let it be noted here that the $4 s \times(2 s+1)$-dimensional matrix $P_{\mu} \tau^{\mu}$ corresponds to $w(P)$ in $\left[{ }^{1}\right]$ if in (B.1) the matrices $e_{a}(1 / 2)$ are replaced by larger ones

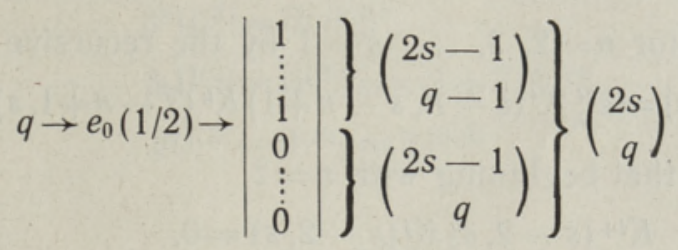

and

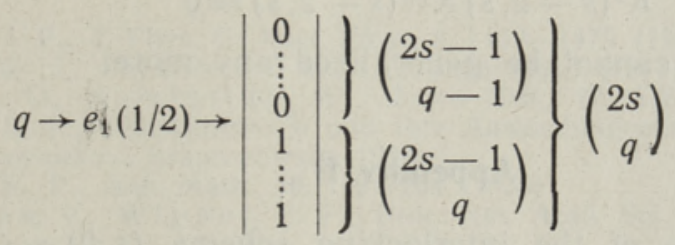

with suitable normalizing factor.

\section{Appendix C}

In order to get the unitary transformation, one needs the following $4 s \times(2 s-1)$-dimensional matrix

$$
t(s)=\left(t_{0}, t_{1}, \ldots, t_{2(s-1)}\right),
$$

where

$$
t_{A}(s)=(2 s)^{-1 / 2}\left(\sqrt{2 s-A+1} \xi_{1, A+1}-\sqrt{A+1} \xi_{2, A+1}\right)
$$

and $A=0,1, \ldots, 2(s-1)$.

The matrix $t$ is a solution of the equations

$$
\begin{gathered}
\tau^{0+} t=0, \\
t+t=I(2 s-1), \\
\tau^{0} \tau^{0+}+t t^{+}=I(4 s) .
\end{gathered}
$$

In addition to (B.3), the following relations are valid

$$
\begin{aligned}
& t^{++} \tau_{\varepsilon}^{i} M^{j}(s)-M^{j}(s-1) t^{+} \tau_{\varepsilon}^{i}=i \varepsilon_{k}^{i j t+} \tau_{\varepsilon}^{k}, \\
& t^{+}\left(\tau_{\varepsilon}^{i} \tau_{\varepsilon}^{j+}-\tau_{\varepsilon}^{j} \tau_{\varepsilon}^{i+}\right) t=-i(2 s+1) s^{-2} \varepsilon_{k}^{i j} M^{k}(s-1), \\
& t^{+}\left(\tau_{\varepsilon}^{\mu} \tau_{\varepsilon}^{v+}-\tilde{\tau}_{\varepsilon}^{v} \tau_{\varepsilon}^{\mu+}\right) \tau^{0}=\varepsilon t^{t+}\left(g^{0 \mu} \tau_{\varepsilon}^{v}-g^{0 v} \tau_{\varepsilon}^{\mu}+(s+1) s^{-1} \varepsilon_{\alpha}^{0 \mu v} \tau_{\varepsilon}^{\alpha}\right) .
\end{aligned}
$$


The relations (B.3), (C.3) show that $t^{+} \tau_{\varepsilon}^{\mu}=\frac{\varepsilon}{s} K^{\mu}$, where $K^{\mu}$ are the matrices introduced by W. J. Hurley $\left[{ }^{2}\right]$, which, in turn, can be expressed by the Clebsch-Gordan coefficients as follows -

$$
\begin{aligned}
& K^{1}=s\{\langle s-1| 1 / 2)(-1 / 2|s\rangle+\langle s-1|-1 / 2)(1 / 2|s\rangle\}, \\
& K^{2}=i s\{-\langle s-1| 1 / 2)(-1 / 2|s\rangle+\langle s-1|-1 / 2)(1 / 2|s\rangle\}, \\
& K^{3}=s\{\langle s-1| 1 / 2)(1 / 2|s\rangle-\langle s-1|-1 / 2)(-1 / 2|s\rangle\} .
\end{aligned}
$$

Here $\left(\sigma|j\rangle_{\alpha p}=(1 / 2, s-1 / 2 ; \sigma \alpha|j p\rangle\right.$ are the Clebsch-Gordan coefficients and $\sigma= \pm 1 / 2, j=s, s-1, \alpha=0,1, \ldots, 2 s-1, p=0,1, \ldots, 2 j$.

Relation (C.3a) may be generalized to arbitrary values of $n$ :

$$
K^{i}(s-n, s) M^{j}(s)-M^{j}(s-n) K^{i}(s-n, s)=i \varepsilon_{k}^{0 i j} K^{k}(s-n, s),
$$

where $K^{i}$ are given for $n=2,3, \ldots, s-1$ by the recursive relation

$$
K^{i}(s-n, s)=\varepsilon_{j k}^{0 i} K^{j}(s-n, s-n+1) K^{k}(s-n+1, s) .
$$

Actually one can see that beginning with $n=2$

$$
\begin{aligned}
& K^{i+}(s-2, s) K^{j}(s-2, s)=0, \\
& K^{i}(s-2, s) K^{j+}(s-2, s)=0
\end{aligned}
$$

and relation (C.3c) cannot be generalized any more.

\section{Appendix D}

For the linearization of the interlocking scheme $(s, 0) \sim(s+1 / 2,1 / 2)$, one needs the following $4(s+1) \times(2 s+1)$-dimensional matrices - ?

$$
\begin{aligned}
& \left(\varrho_{\varepsilon}^{0}(s)\right)_{q}=t_{q}(s+1), \\
& \left(\varrho_{\varepsilon}^{1}(s)\right)_{q}=\varepsilon(2(s+1))^{-1 / 2}\left(-\sqrt{q+1} \xi_{1, q+2}(s+1)+\sqrt{2 s-q+1} \xi_{2 q}(s+1)\right), \\
& \left(\varrho_{\varepsilon}^{2}(s)\right)_{q}=i \varepsilon(2(s+1))^{-1 / 2}\left(\sqrt{q+1} \xi_{1, q+2}(s+1)+\sqrt{2 s-q+1} \xi_{2 q}(s+1)\right), \\
& \left(\varrho_{\varepsilon}^{3}(s)\right)_{q}=\varepsilon(2(s+1))^{-1 / 2}\left(\sqrt{2 s-q+1} \xi_{1, q+1}(s+1)+\sqrt{q+1} \xi_{2, q+1}(s+1)\right) \\
& (q=0,1, \ldots, 2 s),
\end{aligned}
$$

which obey the relation

$$
\tilde{Q}_{\varepsilon}^{\mu+} Q_{\varepsilon}^{v}=g^{\mu \nu}+i(s+1)^{-1} M_{\varepsilon}^{\mu v} .
$$

\section{Appendix E}

The Kemmer-Duffin spin- 0 algebra $\left[{ }^{12,13}\right]$, determined by the relations

$$
\begin{gathered}
\beta_{\mu} \beta_{v} \beta_{\sigma}+\beta_{\sigma} \beta_{v} \beta_{\mu}=g_{\mu v} \beta_{\sigma}+g_{v \sigma} \beta_{\mu}, \\
\beta_{\mu} \beta_{v} \beta_{\sigma}=g_{\mu v} \beta_{5} \beta_{\sigma}+g_{v \sigma} \beta_{\mu} \beta_{5},
\end{gathered}
$$

can be realized by the matrices

$$
\left(\beta_{\mu}\right)_{B}^{A}=g_{\mu}^{A} g_{B 5}+g_{5}^{A} g_{B \mu},
$$

$$
\beta_{5}=g_{\mu \mu} g_{v v} \beta_{\mu} \beta_{\mu} \beta_{v} \beta_{v}, \quad \mu \neq v .
$$




\section{Here the metrics}

$$
g_{A B}=(\bar{g})_{B}^{A}=\left\{\begin{array}{l}
g_{\mu v} \\
1, A=B=5 \\
0, A \neq B
\end{array}\right.
$$

has been used.

The projection operators are

$$
\Pi^{(\Xi)}=(1+\Xi \bar{g}) / 2, \quad \Xi= \pm 1
$$

and they satisfy the relations

$$
\begin{aligned}
& \beta^{0} \Pi^{(\Xi)}=\Pi^{(\Xi)} \beta^{0}=(1 \dashv-\Xi) \beta^{0} / 2, \\
& \beta^{k} \Pi^{(\Xi)}=\Pi^{(-\Xi)} \beta^{k}, \\
& \beta_{5} \Pi^{(\Xi)}=\Pi^{(\Xi)} \beta_{5}=(1+\Xi) \beta_{5} / 2, \\
& \bar{g} \Pi^{(\Xi)}=\Pi^{(\Xi)} \bar{g}=\Xi \Pi^{(\Xi)} .
\end{aligned}
$$

\section{REFERENCES}

1. G a z e a u, J. P., J. Phys. G: Nucl. Phys., 6, 1459-1475 (1980).

2. Hurley, W. J., Phys. Rev., D 4, № 12, 3605-3616 (1971).

3. B a rut, A. O., Samiulla h, M., Nuovo Cim., 17, 876-880 (1960).

4. Wigner, E. P., Gruppentheorie und Ihre Anwendungen auf die Quantegmechanik der Atomspekter, Braunschweig, 1931.

5. Wi g n e r, E. P., Ann. Math., 40, 149-204 (1939).

6. B a rg m a n n, V., W i g n e r, E. P., Proc. Nat. Acad. Sci. USA, 34, № 5, 211-223 (1948).

7. Foldy, L. L., Phys. Rev., 102, 568-581 (1956).

8. S h a w, R., Nuovo Cim., 33, № 4, 1074-1090 (1964).

9. H a rish-Chandra, Phys. Rev., 71, 793-805 (1947).

10. B h a b h a, H. J., Rev. Mod. Phys., 17, 200-216 (1945);

W il d, E., Proc. Roy Soc. (London), A191, 253-268 (1947);

Cors o n, E. M.; Introduction to Tensors, Spinors and Relativistic Wave-Equations, Blackie \& Son, London, 1953;

Гельфанд И. М., Минл ос Р. А., Шап и о о. Я., Представления группы вращений и группы Лоренца, М., Физматгиз, 1958.

11. Л о йд е Р.-К., Изв. АН ЭССР, Физ. Матем., 22, № 3, 317-319 (1973).

12. D u f f i n, R. J., Phys. Rev., 54, 1114 (1938).

13. Kemmer, N., Proc. Roy. Soc., A173, 91-116 (1939).

14. Hurley, W. J., Phys. Rev., D10, 1185-1200 (1974).

15. Samiullah, M., Khalili, K. G., Preprint IC/74/104, Miramare-Trieste, 1974.

\section{Acadeny of Sciences of the Estonian SSR, Institute of Physics}

\section{Tallinn Polytechnic Institute}

\section{KLEIN-GORDIONI VORRANDI LINEARISEERIMISEST MITTETRADITSIOONILISTE MEETODITEGA}

On vaadeldud kaht mittetraditsioonilist meetodit Klein-Gordoni vôrrandi lineariseerimiseks, kusjuures on lähtutud nendest Poincaré rühma taandumatutest esitustest, mis kirjeldavad nullist erineva seisumassi ja suvalise spinniga osakesi. Esimesena on vaadeldud lineariseerimist üldiselt ristkülikukujuliste, vôimalikult minimaalse dimensiooniga maatriksitega. On näidatud, et sel juhul on Gazeau tu'etatud nn. üldistatud Diraci vōrrand ekvivalentne Hurley vōrrandiga. 
- Teine meetod pöhineb Kemmer-Duffini 0 -spinniga $\beta$-algebra omaduste kasutamisel. On konstrueeritud invariantne lagranžiaan ja esitatud võrrand energia operaatori oma. väärtuste leidmiseks minimaalse elektromagnetilise interaktsiooni korral.

P. СААР, Р.-К. ЛОИДЕ

\section{ЛИНЕАРИЗАЦИЯ УРАВНЕНИЯ КЛЕИНА-ГОРДОНА НЕТРАДИЦИОННЫМИ МЕТОДАМИ}

Рассмотрены два нетрадиционных метода линеаризации уравнения Клейна-Гордона. 3а исходные приняты те неприводимые представления группы Пуанкаре, которые описывают частицы с ненулевой массой покоя и произвольным спином. Первый метод реализуется с помощью прямоугольных матриц наименьших размерностей. Показано, что в этом случае т. н. обобщенное уравнение Дирака, выведенное Дж. П. Газо, эквивалентно уравнению Хэли. Второй метод линеаризации основывается на $\beta$-алгебре Кеммера-Дэффина с нулевым спином. При наличии минимального электромагнитного взаимодействия сконструирован инвариантный лагранжиан и выведено уравнение для собственных значений оператора энергии. 